\title{
A FIXED POINT CRITERION FOR LINEAR REDUCTIVITY
}

\author{
PETER NORMAN
}

ABSTRACT. Let $G$ be a linear algebraic group over an algebraically closed field. If for all actions of $G$ on smooth schemes, the fixeld point scheme is smooth, then $G$ is linearly reductive under either of the additional assumptions: (a) the ground field is characteristic zero, or (b) $G$ is connected, reduced, and solvable.

Let $K$ be an algebraically closed field and $G$ a linear algebraic group over $K$. Say $G$ has the smooth fixed point property if for all actions of $G$ on a smooth scheme, the fixed point scheme is also smooth. Fogarty [1] has shown that if $G$ is linearly reductive, then $G$ has the smooth fixed point property. One can ask the converse question: If $G$ is not linearly reductive, is there an action of $G$ on a smooth scheme with a nonsmooth fixed point scheme? In this note we show how to construct such an action for any $G$ that is a split extension by a unipotent subgroup. This gives an affirmative answer to the question for any class of groups where $G$ being not linearly reductive implies $G$ is a split extension by a unipotent subgroup. In particular this includes all groups of characteristic zero and in arbitrary characteristic connected reduced solvable groups.

Let $G$ be a linear algebraic group over $K$ which is a split extension by the unipotent subgroup $U$. We first show that we may assume that $U$ is the direct sum of copies of the additive group. If $G$ modulo a normal subgroup has an action with a nonsmooth fixed point scheme, then certainly $G$ does. Using this we can replace $G$ by $G$ modulo the commutator subgroup of $U$, and hence we may assume $G$ is commutative. In characteristic zero this already implies $U$ is the direct sum of copies of the additive group. In characteristic $p$ there are truncated Witt groups; however, if we take $G$ modulo $p \cdot U$ we may assume $G$ is the direct sum of additive groups by a theorem of Serre [2].

We now construct an action of $G$ on affine space with a nonreduced fixed point scheme. Using our assumption that $G$ is a split extension, we 
pick a section for the exact sequence

$$
0 \rightarrow U \rightarrow G \rightarrow G / U \rightarrow 0
$$

Using this section we write elements of $G$ as ordered pairs $(u, t)$ with $u \in U$ and $t \in G / U$. Let $\rho: G / U \rightarrow$ Aut $(U)$ give the action of $G / U$ on $U$. The multiplication in $G$ is given by

$$
(u, t) \cdot\left(u^{\prime}, t^{\prime}\right)=\left(u+\rho(t) \cdot u^{\prime}, t \cdot t^{\prime}\right) .
$$

Let $X=U \times \operatorname{spec} K[x]=\operatorname{spec} K\left[y_{1} \cdots y_{n}, x\right]$. In terms of these coordinates on $U$, let $\rho(t)$ be given by the matrix $\left(\rho_{i j}(t)\right)$. The action of $G$ on $X$ is given by

$$
(u, t):\left\{\begin{array}{l}
x \mapsto x, \\
y_{i} \mapsto \Sigma_{j} \rho_{i j}(t) y_{j}+u_{i} x^{2}, \quad i=1, \cdots, n,
\end{array}\right.
$$

where $u=\left(u_{1} u_{2} \cdots u_{n}\right)$. We now verify that this is an action. Let $\left(u^{\prime}, t^{\prime}\right)=$ $\left(u_{1}^{\prime}, u_{2}^{\prime}, \cdots, u_{n}^{\prime}, t\right)$ be another element of $G$. Now $x$ is fixed so there is nothing to do with $x$.

$$
\begin{gathered}
(u, t): y_{i} \mapsto \sum_{j} \rho_{i j}(t) y_{j}+u_{i} x^{2} \\
\left(u^{\prime}, t^{\prime}\right): \sum \rho_{i j}(t) y_{j}+u_{i} x^{2} \mapsto \sum_{j} \rho_{i j}(t)\left(\sum_{k} \rho_{j k}\left(t^{\prime}\right) y_{k}+u_{j}^{\prime} x^{2}\right) \\
+u_{i} x^{2} \sum_{k} \rho_{i k}\left(t \cdot t^{\prime}\right) y_{k}+\left(\sum_{j} \rho_{i j}(t) \cdot u_{j}^{\prime}+u_{i}\right) \cdot x^{2} .
\end{gathered}
$$

But this is also the result of $\left(u+\rho(t) \cdot u^{\prime}, t \cdot t^{\prime}\right)=(u, t) \cdot\left(u^{\prime}, t^{\prime}\right)$ acting on $\left(x, y_{1}, \cdots, y_{n}\right)$.

Now $X$ is affine $n+1$ space and hence smooth. On the other hand, the fixed point scheme of the action by $G$ is defined by the ideal $I$ generated by all the elements of the form $g r-r$ for $r \in K\left[y_{1}, \cdots, y_{n}, x\right]$ and $g \in G$. By setting $t$ to be the identity and $u_{1}=1$ we see that $x^{2} \in I$. But it is clear that $x \notin I$ and so the fixed point scheme is not reduced.

\section{BIBLIOGRA PHY}

1. J. Fogarty, Fixed point schemes, Amer. J. Math. 95 (1973)。

2. J.-P. Serre, Groupes algébriques et corps de classes, Publ. Inst. Math. Univ. Nancago, VII, Actualités Sci. Indust., no. 1264, Hermann, Paris, 1959. MR 21 \#1973; erratum, 30, p. 1200. 